\title{
Literacia em saúde e utilização de serviços
}

Paula Broeiro*

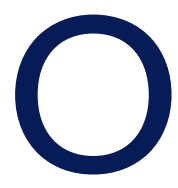

problema cíclico da sobrelotação dos serviços de urgência durante o período epidémico de gripe evidencia a fragilidade do Serviço Nacional de Saúde. As recomendações das autoridades de saúde veiculadas pelos media centram-se na utilização dos serviços e raras vezes na benignidade da situação clínica e nos autocuidados. Como cidadã e interveniente no processo de cuidar incomoda-me a distância emocional entre os decisores e a vivência das pessoas que adoecem.

As pessoas que utilizam mal os serviços, recorrendo inapropriadamente às urgências hospitalares, fazem-no seguramente por uma multiplicidade de razões: têm medo perante as situações graves e mortes noticiadas nos órgãos de comunicação social, desconhecem os autocuidados e/ou não têm recursos de saúde, nomeadamente acessibilidade aos cuidados de saúde primários (e.g., não têm médico de família ou têm médicos de família sobrecarregados). As pessoas que usam mal os serviços não o fazem porque querem consumir recursos de saúde, mas porque estão em sofrimento físico e social! É, pois, uma questão que merece reflexão profunda, não apenas sobre a frieza dos números, mas numa perspetiva ecossocial e narrativa de nos colocarmos na pele do outro, com a pele do outro.

Num exercício de reflexão concetual em saúde pública o que se realça são: os determinantes sociais de saúde e a literacia em saúde (LS). ${ }^{1}$ Centro-me na LS, que é uma expressão frequentemente utilizada sem a devida integração da complexidade que o conceito comporta. A LS é reconhecida como um conceito de importância crítica em saúde pública. ${ }^{2}$ Na sociedade moderna, a complexidade dos sistemas de saúde leva as pessoas a ter que tomar decisões em relação à doença, à prevenção e à promoção da saúde, considerando-se a LS um fator que pode contribuir para desigualdades em saúde. ${ }^{1-2}$ A LS é definida como o grau com que cada

*Directora da Revista Portuguesa de Medicina Geral e Familiar indivíduo tem capacidade de obter, comunicar, processar e compreender as informações, bem como utilizar serviços de saúde de forma a tomar decisões apropriadas em saúde. ${ }^{3-9}$ Inclui a capacidade de interpretar documentos, ler e escrever (literacia impressa), usar informação quantitativa (numeracia) e falar e ouvir com eficácia (literacia oral). ${ }^{5-6} \mathrm{O}$ modelo concetual de LS combina competências necessárias ao processo de aceder, compreender, avaliar e aplicar a informação relacionada com saúde. ${ }^{7}$ Aceder refere-se à capacidade de procurar, encontrar e obter informação; compreender corresponde à capacidade de entender a informação; avaliar descreve a capacidade de interpretar, filtrar e julgar a informação; e aplicar refere-se à capacidade de comunicar, usar a informação e tomar a decisão de manter e melhorar a saúde. ${ }^{?}$

O conceito de LS associa-se frequentemente a capacitação e educação, sendo a educação o preditor de saúde mais forte..$^{10-11}$ Diversas intervenções em saúde fracassaram por se concentrarem na educação e na comunicação em saúde, numa perspetiva individual, negligenciando a importância das condições sociais, culturais, económicas e comunitárias. ${ }^{10}$ Os níveis de LS variam desde um nível básico/funcional (a capacidade de entender a informação sobre saúde) a níveis elevados (autocontrolo, pensamento crítico e reflexão). ${ }^{10} \mathrm{~A}$ LS não conduz automaticamente a capacitação porque esta depende de outras dimensões como motivação, confiança, autoeficácia e tomada de decisão partilhada. ${ }^{2,10}$ Como é que pessoas com necessidades básicas insatisfeitas, como alimentação, abrigo, segurança, senso de dignidade, igualdade e pertença entre outros, superam os efeitos negativos de comportamentos de risco (e.g., fumar ou beber álcool)? Isto poderá explicar o insucesso e o desperdício de recursos quando a ênfase é centrada em comportamentos ${ }^{10}$ e não no contexto.

Nas famílias, a LS está relacionada com o nível educacional (especialmente da mãe) e, ao longo de décadas, estabelecida como importante preditor de resul- 
tados de saúde. ${ }^{11}$ A educação tem sido um dos principais mediadores de resultados em saúde, em contextos ecossociais desfavoráveis. ${ }^{11}$ Em diversos estudos, o nível de instrução (o número de anos de escolaridade) tem sido considerado o indicador-chave de LS, no entanto, não se relacionando de forma linear. ${ }^{11} \mathrm{~A}$ educação contribui para o desenvolvimento de uma variedade de capacidades cognitivas: resolução de problemas, pensamento crítico e autocontrolo. ${ }^{7,11}$ A educação desenvolve, ainda, a capacidade de interpretar a informação (e.g., rótulos de medicamentos), de resolver problemas e aumenta o potencial de controlar eventos de saúde.9,11 Mas também o "lugar" e o efeito da vizinhança são importantes para a saúde (efeitos contextuais). ${ }^{1,7,11}$ Em saúde, os indivíduos com mais educação beneficiam dos ambientes nos quais tendem a viver, estudar e trabalhar. ${ }^{7,11}$

A importância da LS para a saúde pública é suportada pelo número crescente de estudos que associam a baixa literacia a piores resultados, ${ }^{2,5,9,11}$ tal como a taxa de mortalidade mediada por fatores intermédios como autocuidado, comportamentos de risco, participação em programas preventivos e adesão à terapêutica., ${ }^{2,11-12}$ Schumacher e colaboradores ${ }^{13}$ realizaram um estudo observacional que analisou a utilização inapropriada dos serviços de urgência e a LS. A maioria dos participantes (89\%) acreditava que a sua condição era uma urgência e que o serviço de urgência era o local certo para tratamento (92\%). ${ }^{13}$ Verificou-se uma utilização mais frequente por doentes com baixa LS, tendo sido esta associação estatisticamente significativa quando comparada com os com adequada LS $(60 \%$ vs $40 \%$, $p<0,001) .{ }^{13}$ Um outro estudo, belga, mais recente, confirma a associação entre a baixa LS e o uso menos eficiente dos serviços de saúde, como o recurso ao atendimento de urgência. ${ }^{2}$ No entanto, na Europa, os resultados de estudos sobre custos em saúde decorrentes do uso inapropriado de serviços são inconsistentes. ${ }^{2}$

A investigação social sugere que o estatuto social desigual cria acesso desigual a recursos, incluindo de saúde, tornando-se um desafio crítico a compreensão do nexo entre fatores como condição social, educação e saúde. ${ }^{1,11}$ Apesar da necessidade dos profissionais de saúde desenvolverem conhecimentos, capacidades e atitudes adequados a pacientes e seus cuidadores com baixa LS, ${ }^{4}$ a questão da literacia não se resume a cuida- dos de saúde. ${ }^{8}$ Requer uma abordagem sistémica que inclua políticas de saúde e de educação e que envolva a sociedade.

A diferente utilização de serviços mobiliza recursos pessoais como qualquer mudança de comportamento. Quando se recomenda a não ida aos serviços de urgência não estaremos a excluir mais uma vez pessoas com baixo nível LS, associados a determinantes sociais de saúde (e.g., trabalho indiferenciado precário)? É, pois, necessária uma investigação ecossocial sobre a utilização dos serviços de saúde. ${ }^{1,11}$

Enquanto Europeus deveríamos zelar pela nossa identidade humanista e contribuir nos nossos lugares para minimizar desigualdades, assegurando a proteção dos direitos humanos, não deixando ninguém para trás.

Em suma, seguir o exemplo de António Guterres enquanto Secretário-Geral das Nações Unidas, de quem transponho algumas das suas ideias-chave:

- Valores: Paz, justiça, dignidade humana, tolerância e solidariedade ${ }^{14}$

- Comunicar melhor, de maneira a que todos entendam $^{15}$

- Focar: mais na entrega e menos no processo, mais nas pessoas que na burocracia ${ }^{15}$

- Cooperar em vez de duplicar, partilhar em vez de competir e assumir responsabilidade coletiva em vez de interesses individuais ${ }^{14}$

- No nosso mundo complexo colocar a parceria no centro da estratégia e reconhecer com humildade o papel essencial de outros atores. ${ }^{15}$

\section{REFERÊNCIAS BIBLIOGRÁFICAS}

1. Kickbusch I, Pelikan JM, Apfel F, Tsouros AG. Health literacy: the solid facts [Internet]. Copenhagen:World Health Organization Regional Office for Europe; 2013. ISBN 9789289000154. Available from: http:// www.euro.who.int/_data/assets/pdf_file/0008/190655/e96854.pdf

2. Vandenbosch J, Van Den Broucke S, Vancorenland S, Avalosse H, Verniest R, Callens M. Health literacy and the use of healthcare services in Belgium. J Epidemiol Community Health. 2016;70(10):1032-8.

3. Somers SA, Mahadevan R. Health literacy implications of the Affordable Care Act [Internet]. Washington, DC: Center for Health Care Strategies; 2010. Available from: http://www.chcs.org/media/Health_Literacy_Implications_of_the_Affordable_Care_Act.pdf

4. Coleman CA, Hudson S, Maine LL. Health literacy practices and educational competencies for health professionals: a consensus study. J Health Commun. 2013;18 Suppl 1:82-102.

5. Musa TH, Ping-min W, Yuepu P. Review: health literacy intervention and 
their consequences. J Public Health Epidemiol. 2015;7(3):71-5.

6. Baker DW. The meaning and the measure of health literacy. J Gen Intern Med. 2006;21(8):878-83.

7. Sørensen K, Van Den Broucke S, Fullam J, Doyle G, Pelikan J, Slonska Z, et al. Health literacy and public health : a systematic review and integration of definitions and models. BMC Public Health. 2012;12:80.

8. Rudd RE. Needed action in health literacy. J Health Psychol. 2013;18 (8):1004-10.

9. Pedro AR, Amaral O, Escoval A. Literacia em saúde, dos dados à ação: tradução, validação e aplicação do European Health Literacy Survey em Portugal [Health literacy, from data to action: translation, validation and application of the European Health Literacy Survey in Portugal] Rev Port Saúde Pública. 2016;34(3):259-75. Portuguese

10. Crondahl K, Karlsson LE. The nexus between health literacy and empowerment: a scoping review. SAGE Open. 2016;6(2).

11. Zimmerman EB, Woolf SH, Haley A. Understanding the relationship between education and health: a review of the evidence and an examination of community perspectives [Internet]. Rockville, MD:Agency for Healthcare Research and Quality; 2014 [updated 2015 Sep]. Available from: https://www.ahrq.gov/professionals/education/curriculumtools/population-health/zimmerman.html

12. Annarumma C. Contextualizing health literacy to health care organizations: exploratory insights. J Health Manag. 2016;18(4):611-24.

13. Schumacher JR, Hall AG, Davis TC, Arnold CL, Bennett RD, Wolf MS, et al. Potentially preventable use of emergency services: the role of low health literacy. Med Care. 2013;51(8):654-8.

14. Guterres A. Challenges and opportunities for United Nations. The Huffington Post [Internet]; 2016 May 11. Available from: http://www.huffingtonpost.com/antanio-guterres/challenges-and-opportunities-forthe-united-nations_b_9796624.html

15. Guterres A. Nations U. Secretary-General-designate António Guterres' remarks to the General Assembly on taking the oath of office [Internet]. Washington, DC: United Nations; 2016. Available from: https:// www.un.org/sg/en/content/sg/speeches/2016-12-12/secretary-general-designate-ant\%C3\%B3nio-guterres-oath-office-speech

\section{ENDEREÇO PARA CORRESPONDÊNCIA}

director@rpmgf.pt 